\title{
Análisis de la accesibilidad física en el transporte público en autobús en la ciudad de Valladolid
}

\author{
Analysis of physical accessibility in public \\ transport by bus in the city of Valladolid
}

\section{Resumen}

La accesibilidad al transporte público es uno de los factores que limitan la integración de las personas con algún tipo de discapacidad en la sociedad. En este trabajo hemos analizado durante seis meses la situación de la cadena de accesibilidad del autobús urbano, contrastando la ficha de toma de datos con expertos de la Universidad de Valladolid, personal técnico y usuarios de Cocemfe Castilla y León y realizando el análisis de las casi 600 paradas de la ciudad de Valladolid, diferenciando entre aspectos críticos y no críticos (aunque recogidos en la legislación) para personas con discapacidad física.

\section{Palabras clave}

Discapacidad, exclusión social, transporte público, accesibilidad universal, personas con discapacidad física.

\section{Abstract}

Accessibility to public transport is one of the factors that limit the integration of people with some type of disability into society. In this paper we have analyzed the situation of the accessibility chain of the city bus for 6 months, contrasting the data collection sheet by experts of the University of Valladolid, technical staff and users of Cocemfe Castilla y Leon and performing the analysis of nearly 600 stops in the city of Valladolid, differentiating between critical and noncritical aspects (although collected in law) for people with physical disabilities.

\section{Keywords}

Disability, social exclusion, public transport, universal design, people with physical disabilities.

\section{Angel Manuel Gento \\ Municio \\ <gento@eii.uva.es> \\ Universidad de Valladolid}

Juan Luis Elorduy González

<jlelorduy@gmail.com>

Universidad de Valladolid
Para citar:
Gento, A.M. y Elorduy, J.L. (20I6):
"Análisis de la accesibilidad física
en el transporte público en autobús
en la ciudad de Valladolid”, Revista
Española de Discapacidad, 4 (I):
I35-I 53.
Doi: <http://dx.doi.org/I0.5569/234O-
5I04.04.0I.08>

Fecha de recepción: I2-02-20I 6

Fecha de aceptación: 30-05-2016 


\section{Introducción}

La Convención Internacional sobre los Derechos de las Personas con Discapacidad (CDPD) (ONU, 2006), en su artículo 2 establece que por "discriminación por motivos de discapacidad se entenderá cualquier distinción, exclusión o restricción por motivos de discapacidad que tenga el propósito o el efecto de obstaculizar o dejar sin efecto el reconocimiento, goce o ejercicio, en igualdad de condiciones, de todos los derechos humanos y libertades fundamentales en los ámbitos político, económico, social, cultural, civil o de otro tipo". Y queda prohibida en los estados que ratifiquen la convención ( 3 de mayo de 2008 en el caso de España).

Esta convención en su artículo 9 relativo a la accesibilidad, señala que: “A fin de que las personas con discapacidad puedan vivir en forma independiente y participar plenamente en todos los aspectos de la vida, los Estados Partes adoptarán medidas pertinentes para asegurar el acceso de las personas con discapacidad, en igualdad de condiciones con las demás, al entorno físico, el transporte, la información y las comunicaciones, incluidos los sistemas y las tecnologías de la información y las comunicaciones, y a otros servicios e instalaciones abiertos al público o de uso público, tanto en zonas urbanas como rurales".

Son numerosos los autores que han relacionado en artículos la discriminación con el concepto de exclusión social en las personas con discapacidad. (Corbacho, 2003; Ferreira, 2008; Díaz Velázquez, 20I0; Huete, 20I2).

"La exclusión social incluye dificultades o barreras en al menos el eje económico (empleo, ingresos, privación), el eje político de la ciudadanía (derechos políticos, educación, vivienda o salud) y el eje de las relaciones sociales (aislamiento, desestructuración familiar)" (Huete, 2013: 8), y "la exclusión social describe la existencia de barreras que hacen difícil o imposible que las personas participen completamente en la sociedad" (Stanley et al., 20 Iо: I). Por otro lado la utilización del transporte público es un elemento facilitador del desarrollo de las capacidades de las personas y un canalizador para su participación en la sociedad.

Por lo tanto las restricciones de movilidad en el transporte pueden ser una causa de exclusión social (Preston y Rajé, 2007; Preston, 2009; Priya y Uteng, 2009). Las personas con discapacidad se identifican como uno de los grupos que pueden sufrir esta exclusión (Barnes y Mercer, 2005; Casas, 2007) y el incorrecto acceso y uso del transporte público como una de las causas (Field y Jette, 2007; Lucas 20I2). Los estudios científicos realizados se han basado en análisis empíricos de las percepciones de los usuarios con dificultades para utilizar el transporte público (personas sin discapacidad y personas con discapacidad). Aarhaug y Elvebakk (2OI 5 ) concluyen que el diseño universal es rentable desde el punto de vista económico y social.

No se han encontrado artículos científicos que analicen las condiciones de accesibilidad en el transporte público para la utilización por parte de una persona con discapacidad física, aunque hay estudios realizados por algunas asociaciones y organismos públicos.

Este trabajo, enmarcado dentro de las líneas de investigación del Máster en Logística de la Universidad de Valladolid, tiene como objetivo extraer conclusiones sobre el estado de la accesibilidad en el transporte público de autobuses en la ciudad de Valladolid, tomando como punto de partida el I Plan Municipal de Accesibilidad (20I I) y el Plan Integral de Movilidad Urbana Ciudad de Valladolid (2005) (www.valladolid.es). 


\section{Accesibilidad y transporte}

El concepto de accesibilidad universal se recoge en el Artículo 2 del Real Decreto Legislativo I/20I3, de 29 de noviembre, por el que se aprueba el Texto Refundido de la Ley General de derechos de las personas con discapacidad y de su inclusión social ${ }^{\mathrm{x}}$, y se define como "la condición que deben cumplir los entornos, procesos, bienes, productos y servicios, así como los objetos, instrumentos, herramientas y dispositivos, para ser comprensibles, utilizables y practicables por todas las personas en condiciones de seguridad y comodidad y de la forma más autónoma y natural posible". Presupone la estrategia de «diseño universal o diseño para todas las personas», y se entiende sin perjuicio de los ajustes razonables que deban adoptarse".

La accesibilidad universal se centra en garantizar la igualdad entre personas con cualquier tipo de discapacidad, ya sea física, mental, intelectual o sensorial para el acceso a cualquier ámbito, ya sea por ejemplo acceso a la vivienda, movilidad en el transporte, movilidad en el entorno, acceso a un puesto de trabajo... incluyendo productos, sistemas de información, comunicación, etc.

La finalidad principal de la accesibilidad universal es garantizar la cadena de accesibilidad, entendiendo como tal todos los elementos que posibilitan que se pueda realizar una determinada actividad en un entorno concreto. Cuando existe algún eslabón de la cadena que no cumple esas condiciones, nos encontramos con las denominadas barreras de accesibilidad (Juncà, 2006; Ararteko, 20I I; Aarhaug y Elvebakk, 20I 5).

La Ley 3/I998, de 24 de junio de accesibilidad y supresión de barreras de Castilla y León ${ }^{2}$ (en adelante Ley 3/I998), considera barrera de accesibilidad a "cualquier obstáculo que impida o limite la autonomía personal”. Éstas

I. BOE, núm. 289, de 3 de diciembre de 2013.

2. BOCYL núm. I23, de I de julio de 1998. se pueden clasificar en cuatro tipos distintos: arquitectónicas (las existentes en la edificación), urbanísticas (las existentes en las vías públicas, así como en los espacios libres de uso público y todos los privados de uso colectivo), de transporte (las que se originan en los medios de transporte e instalaciones complementarias) y de comunicación (las que dificultan o imposibilitan la recepción de mensajes a través de los medios, sistemas y técnicas de comunicación).

Para la realización de este estudio es importante considerar no únicamente el concepto de accesibilidad universal, sino el grado de autonomía que puede tener una persona con alguna discapacidad o alguna limitación para poder acceder a un determinado producto/ servicio o el acceso a un medio de transporte público.

El artículo 2.I de la Ley 39/2006, de I4 de diciembre, de Promoción de la Autonomía Personal y Atención a las personas en situación de dependencia ${ }^{3}$ define el concepto de autonomía como "la capacidad de controlar, afrontar y tomar por propia iniciativa, decisiones personales acerca de cómo vivir de acuerdo con las normas y preferencias propias, así como de desarrollar las actividades básicas de la vida diaria". El artículo 2.3 de la citada Ley define las actividades básicas de la vida diaria (ABVD) como "las actividades más elementales e imprescindibles sin las que una persona no podría subsistir o desenvolverse de manera independiente, con un mínimo de autonomía, como son: el cuidado personal (asearse, vestirse, desnudarse, poder ir solo al servicio, poder quedarse solo durante la noche...), las actividades domésticas básicas, la movilidad esencial (dentro del hogar), reconocer personas y objetos, orientarse, entender y ejecutar órdenes o tareas sencillas", mientras que las actividades instrumentales de la vida diaria (AIVD) son “actividades más complejas que las ABVD y su realización requiere de un mayor nivel de autonomía personal. Se asocian a tareas que implican la capacidad de tomar decisiones e implican interacciones más difíciles con el

3. BOE, núm. 299, de I 5 de diciembre de 2006. 
medio. En esta categoría se incluyen tareas domésticas, de movilidad, de administración del hogar y de la propiedad, como por ejemplo poder utilizar el teléfono, acordarse de tomar la medicación, cortarse las uñas de los pies, subir una serie de escalones, coger un autobús, un metro o un taxi, preparar la propia comida, comprar lo que se necesita para vivir, realizar actividades domésticas básicas (fregar los platos, hacer la cama, etc.), poder pasear, ir al médico, hacer papeleos y administrar el propio dinero, entre otras" (Imserso, 2004: 23).

Según esta clasificación, utilizar un transporte público no se considera una ABVD sino que se considera una AIVD, para lo cual se necesita un nivel de autonomía mayor.

Otro concepto necesario para comprender el estudio realizado es la clasificación de los espacios, instalaciones, edificaciones o servicios según su nivel de accesibilidad en adaptados, practicables y convertibles. La Ley 3/1998 indica que un espacio, instalación o servicio se considera adaptado si se ajusta a los requerimientos funcionales y dimensiones que garanticen su utilización autónoma y con comodidad por las personas con limitación, movilidad o comunicación reducida. Esta misma Ley considera un espacio, instalación o servicio como practicable cuando, sin ajustarse a todos los requerimientos que lo consideren como adaptado, no impide su utilización de forma autónoma a las personas con limitación o movilidad o comunicación reducida. Esta clasificación es aplicable a los itinerarios peatonales, que se definen como todos aquellos espacios públicos destinados al tránsito de peatones o mixto de peatones y vehículos.

\subsection{Evolución legislativa}

La Constitución Española de $1978^{4}$ en su Artículo 9.2 refleja que "corresponde a los poderes públicos promover las condiciones para que la libertad y la igualdad del individuo $\mathrm{y}$ de los grupos en que se integra sean reales y

4. BOE, núm. 3II, de 29 de diciembre de 1978. efectivas; remover los obstáculos que impidan o dificulten su plenitud y facilitar la participación de todos los ciudadanos en la vida política, económica, cultural y social".

La Ley I3/I982, de 7 de abril, de integración social de los minusválidos ${ }^{5}$, conocida coloquialmente con la abreviatura LISMI fue un primer paso al incluir las condiciones urbanísticas y arquitectónicas para asegurar la accesibilidad.

El siguiente paso fue la Ley $5 \mathrm{I} / 2003$, de 2 de diciembre, de igualdad de oportunidades, no discriminación y accesibilidad universal de las personas con discapacidad ${ }^{6}$, denominada LIONDAU, que complementaba a la anterior impulsando la igualdad de las personas con discapacidad, fomentando la lucha contra la discriminación y la accesibilidad universal. En esa ley se recogen los conceptos de accesibilidad universal y diseño universal así como medidas de fomento de la accesibilidad y la no discriminación.

Por primera vez, en esta ley, se mencionaba la obligatoriedad por parte del gobierno de establecer unas condiciones básicas para el acceso y utilización de los medios de transporte para personas con discapacidad fijando una serie de plazos para su elaboración y cumplimiento desde la entrada en vigor de la Ley.

En el año 2007 se aprobaron las condiciones específicas en cuanto a transporte se refiere por el Real Decreto I 544/2007, de 23 de noviembre, por el que se regulan las condiciones básicas de accesibilidad y no discriminación para el acceso y utilización de los modos de transporte para personas con discapacidad ${ }^{7}$ (en adelante Real Decreto I 544/2007).

En este texto legislativo (concretamente en sus anexos) se detallan las condiciones básicas de accesibilidad y no discriminación para su utilización por las personas con discapacidad

\footnotetext{
5. BOE, núm. I03, de 30 de abril de 1982.

6. BOE, núm. 289 , de 3 de diciembre de 2003.

7. BOE, núm. 290, de 4 de diciembre de 2007.
} 
para los diferentes modos de transporte, en concreto: transporte ferroviario, marítimo, aéreo, por carretera, en autobús urbano y suburbano, ferrocarril metropolitano, taxi y servicios de transporte especial.

La Ley 26/20I I, de I de agosto, de adaptación normativa a la Convención Internacional sobre los Derechos de las Personas con Discapacidad ${ }^{8}$ ratifica en el estado Español dicha convención e incorpora como principios generales, entre otros, la autonomía individual y la independencia de cada ser humano. Esta Ley establece unos nuevos plazos para el cumplimiento, en materia de condiciones básicas de accesibilidad y no discriminación para el acceso y utilización de los medios de transporte.

Posteriormente se ha desarrollado el Real Decreto I 276/20I I, de adaptación normativa a la Convención Internacional sobre los derechos de las personas con discapacidad ${ }^{9}$, en la que se plantea la obligatoriedad de elaborar Planes de Accesibilidad en todos los sectores del transporte en grandes infraestructuras y servicios así como facilitar el acceso a los transportes y elementos básicos de información de accesibilidad en pequeñas infraestructuras y servicios.

El último avance es el Real Decreto Legislativo I/20I3, de 29 de noviembre, por el que se aprueba el Texto Refundido de la Ley General de derechos de las personas con discapacidad y de su inclusión social ${ }^{\text {1o }}$. Este texto armoniza mucha de la legislación existente e incluye el principio de promover la autonomía personal, la accesibilidad universal, el acceso al empleo y la vida independiente.

En esta ley se definen los conceptos de vida independiente, accesibilidad universal y diseño universal y se especifica que deberá cumplirse en materia de transporte.

Los plazos establecidos en este Real Decreto Legislativo, en cuanto a acceso y utilización de

8. BOE, núm. I 84 , de 2 de agosto de 20 I I.

9. BOE, núm. 224, de I7 de septiembre de 20 II.

Io. BOE, núm. 289, de 3 de diciembre de 2013. los medios de transporte y espacios públicos urbanizados y edificaciones son el cuatro de diciembre de 20I0, para infraestructuras y material de transporte nuevos y el cuatro de diciembre de 20I7, para infraestructuras y material de transporte existentes que sean susceptibles de ajustes razonables.

\subsection{Percepción de las personas con discapacidad}

Castilla y León es la quinta comunidad de España por número de personas con algún tipo de discapacidad, un 6,05\% (I 52.557 personas) del total de la población, dato por encima de la media nacional que es un 5,44\% (Imserso, 20I3).

En la Encuesta sobre Discapacidades, Autonomía personal y situaciones de Dependencia (INE, 2008), con una muestra de 27 I.000 personas, se investigó la percepción subjetiva de las personas acerca de su discapacidad, entendida como una limitación a la hora de realizar alguna actividad.

El vehículo particular y el transporte público son los medios más utilizados para los desplazamientos de las personas con discapacidad ( $58,3 \%$ y $42,5 \%$ respectivamente), el transporte especial (ambulancias y autocares o microbuses destinados a este colectivo) representa el 9,2 \%, mientras que un $7,3 \%$ no utiliza ningún medio.

En ese estudio se refleja que el 58, I $4 \%$ de personas con discapacidad manifiestan alguna dificultad para utilizar el transporte público (el $55,08 \%$ en la Comunidad de Castilla y León). Los problemas estudiados en la accesibilidad de los transportes públicos para personas que poseen algún grado de discapacidad se presentan en el Gráfico I.

El principal obstáculo para las personas que poseen algún grado de discapacidad en el transporte público es subir o bajar del vehículo, tanto a nivel Nacional (40,7 I \%) como en Castilla y León $(38,66 \%$ ), seguido del acceso 
Gráfico 1. Porcentaje de personas con discapacidad que manifiestan dificultad para el uso del transporte público (\%)

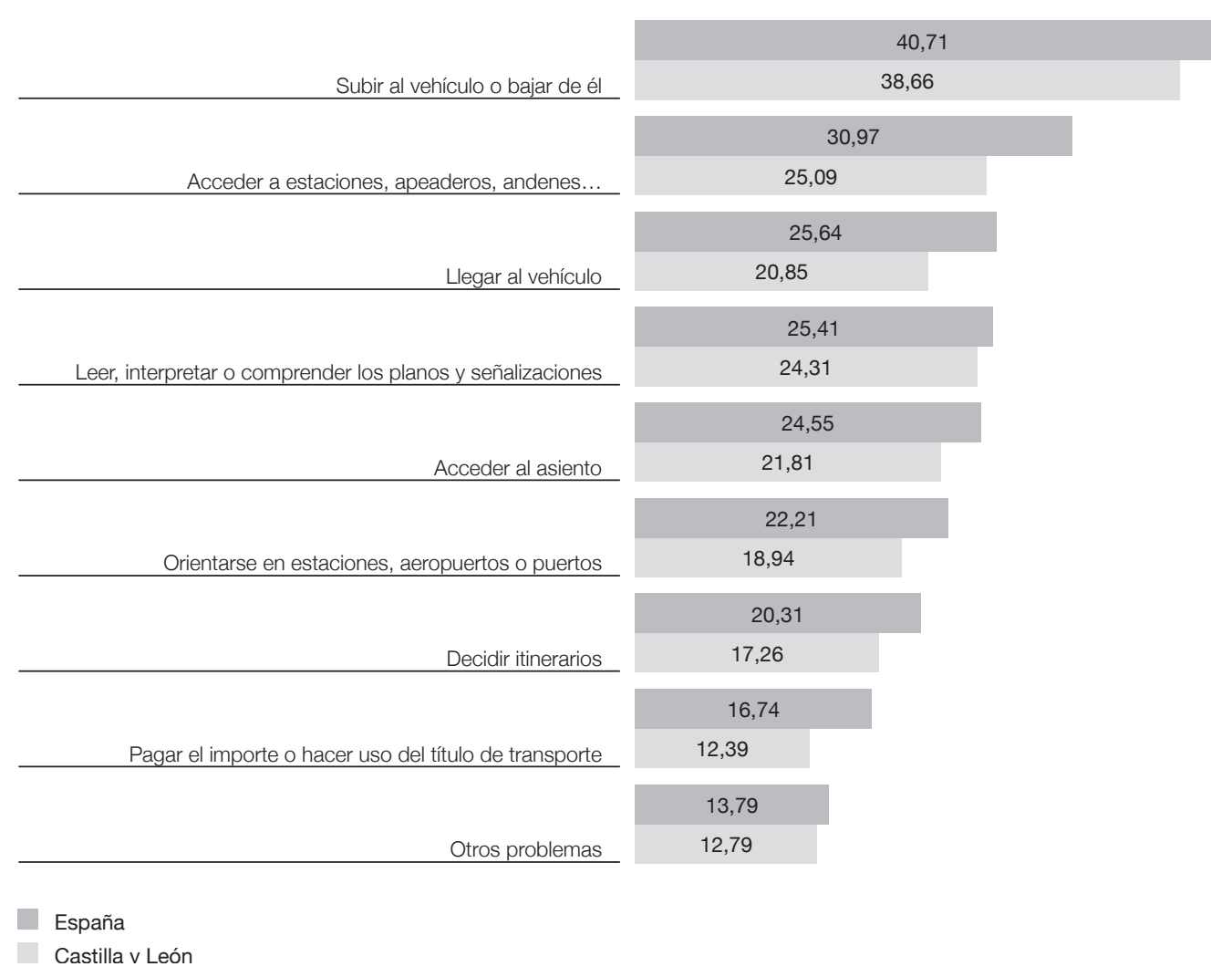

Fuente: EDAD-INE, 2008.

a estaciones, apeaderos y andenes, $(30,97 \%$ y $25,09 \%$ respectivamente), siendo el aspecto que menos problemática presenta el acceso para pagar el importe o hacer uso del título del transporte ( $6,74 \%$ y I $2,39 \%$ ).

\section{Valladolid}

Valladolid es la capital de la provincia del mismo nombre y de la comunidad autónoma de Castilla y León.
Según los últimos datos del Instituto Nacional de Estadística ( I de enero de 20I 5), en Valladolid se encuentran censados 303.905 habitantes, siendo el decimotercer municipio español por número de habitantes.

Considerando el área metropolitana ( 23 municipios) su influencia puede alcanzar a 4II.508 habitantes (INE, 20I5).

Las últimas estadísticas de la Base de datos Estatal de personas con discapacidad indican que el 5,49\% de la población de la provincia de Valladolid tienen consideración de persona con discapacidad 
La superficie del municipio de Valladolid es de I97,7 km², según los datos extraídos de su web municipal.

La empresa encargada de la gestión y explotación de los autobuses urbanos de Valladolid es Autobuses Urbanos de Valladolid S.A., Auvasa, desde I.982 hasta la actualidad.

Según los últimos datos proporcionados en el año 2015 por la empresa en su página web (www.auvasa.es) y el Ayuntamiento de Valladolid, podemos caracterizar a AUVASA por los indicadores de la Tabla I:

Tabla 1. Caracterización Auvasa
\begin{tabular}{|l|l|}
\hline Número de viajeros 2014 & 25.800 .480 \\
\hline Número de vehículos & 150 \\
\hline Antigüedad media de la flota & 11,69 años \\
\hline Líneas ordinarias & 23 \\
\hline Líneas servicios nocturnos & 5 \\
\hline Líneas a polígonos Industriales & 9 \\
\hline Líneas servicios matinales & 7 \\
\hline Líneas fútbol & 6 \\
\hline Líneas especiales & 3 \\
\hline Paradas & 581 \\
\hline
\end{tabular}

Fuente: Auvasa.

Todos los vehículos de Auvasa son de Clase $\mathrm{I}^{\mathrm{II}}$ : vehículos provistos de zonas para viajeros de pie que permiten la circulación frecuente de los pasajeros, con capacidad superior a 22 viajeros, además del conductor.

Analizando los datos disponibles para la flota de autobuses de Auvasa en el acceso al medio de transporte se obtienen los siguientes resultados:

- Autobuses de piso bajo ( $100 \%$ ): Poseen una superficie llana sin escalones y un sistema de inclinación (kneeling), que permite

I I. En el Real Decreto I 544/2007, se detallan las condiciones básicas de accesibilidad que tiene que cumplir un autobús de este tipo. bascular el vehículo para facilitar el acceso de los pasajeros al mismo. Toda la flota de autobuses es de piso bajo.

- Autobuses con rampa de acceso para silla de ruedas; se trata de una rampa eléctrica y/o manual plegable situada en la puerta posterior de dos hojas del vehículo. Un total de 86 vehículos $(57 \%)$ poseen rampa de acceso.

La asignación de los vehículos para cubrir las diferentes líneas lo realiza la empresa según la demanda, pudiéndose dar diferentes casuísticas: que el mismo vehículo se utilice siempre y exclusivamente para cubrir la misma línea, otros que rotan según la línea y el horario, y algunos utilizados como refuerzo.

En cualquier caso, desde Auvasa se indica que cualquier persona que posea una discapacidad y que para el acceso al mismo necesite la utilización de la rampa, puede ponerse en contacto con la empresa indicando la línea y horario en la que desea coger el autobús, para así poder planificar dicho recorrido con el autobús adecuado, en el caso de que en esa línea no se estuviera utilizando un vehículo con rampa de acceso.

\section{Metodología}

El objetivo de este análisis ha sido conocer el grado de cumplimiento de la accesibilidad para una persona con discapacidad física al transporte público de autobús en la ciudad de Valladolid, pero no desde el cumplimiento estricto de la normativa sino desde el punto de vista de la autonomía de una persona con discapacidad física.

El estudio es extrapolable a las personas con movilidad reducida cuyo concepto alcanza a cualquier persona con dificultades para desplazarse y para poder utilizar de manera autónoma y funcional los sistemas de transporte, 
bien sea esta merma en la capacidad de desplazarse transitoria o bien provocada por una discapacidad física, psíquica o sensorial, que a su vez puede sobrevenir como consecuencia de la edad o por otras razones.

\subsection{Diseño de cuestionario}

El primer paso ha sido el estudio y análisis de toda la normativa y legislación de referencia en relación con la accesibilidad en los medios de transporte con el objeto de extraer los requisitos a incluir en la ficha de análisis.

Para el diseño de la ficha, se establecieron cuatro criterios generales:

I. Sencilla, fácil de manejar.

2. Comprensible; fácil de entender.

3. Esquemática; que resuma todos los aspectos a valorar.

4. Objetiva, obtención de información concreta no sujeta a diversas interpretaciones.

Atendiendo a estos criterios y a la normativa analizada se elaboró la ficha estructurada en tres partes:

1. Identificación: En esta parte se han incluido los datos que hacen que una infraestructura (en este estudio la parada) esté identificada por completo. Se reflejan datos como número de la parada, dirección y líneas que pasan por la misma.

2. Análisis de requisitos: Este apartado está dividido en dos tipos: críticos y no críticos, definidos en base a las diferentes reuniones realizadas con usuarios y entidades del sector de la discapacidad.

Los requisitos críticos son aquellos que se deben cumplir siempre para que una persona que posee una discapacidad física pueda acceder de manera autónoma a la infraestructura (parada de autobús).
Los requisitos no críticos son aquellos que, aunque también son importantes para la accesibilidad y regulados en la normativa vigente, podrían superarse por personas con discapacidad física ya que no limitan significativamente su autonomía.

En ambos tipos de requisitos, se incluyen los siguientes puntos determinados por la cadena de la accesibilidad.

- Itinerario: Refiriéndonos al trayecto que tiene que recorrer cualquier usuario de transporte público para llegar a la estación, apeadero, parada de autobús o parada de taxis, incluyendo el espacio de la parada en sí.

- Equipamiento o instalación: Hace referencia a los espacios interiores y sus elementos existentes en edificios públicos destinados a transporte. Estamos hablando de, por ejemplo, los aseos, mostradores, mobiliario, etc., existentes en estaciones de tren, estaciones autobuses o cualquier edificación auxiliar.

- Medio: Aludiendo tanto a las condiciones que se tienen que dar en el interior del mismo, como a las características que tienen que cumplir para que sea posible el acceso al mismo.

La distribución total de los 23 requisitos identificados por tipo se refleja en la Tabla 2.

\begin{tabular}{|c|c|c|}
\hline & $\begin{array}{c}\text { Acceso a } \\
\text { Parada (A) }\end{array}$ & Parada (P) \\
\hline CRÍTICOS (C) & 5 & 7 \\
\hline NO CRITICOS (NC) & 2 & 9 \\
\hline
\end{tabular}

Fuente: elaboración propia.

Cada uno de los requisitos puede ser contestado como Si/No/No Aplica y 


\begin{tabular}{|c|c|c|}
\hline Rango & Título & $\begin{array}{l}\text { Fecha de } \\
\text { entrada en } \\
\text { vigor }\end{array}$ \\
\hline \multirow{4}{*}{ Estatal } & $\begin{array}{l}\text { Real Decreto 1544/2007, de } 23 \text { de noviembre, por el que se regulan las } \\
\text { condiciones básicas de accesibilidad y no discriminación para el acceso y } \\
\text { utilización de los modos de transporte para personas con discapacidad }\end{array}$ & $05 / 12 / 2007$ \\
\hline & $\begin{array}{l}\text { Real Decreto 314/2006, de } 17 \text { de marzo, por el que se aprueba el Código } \\
\text { Técnico de la Edificación y modificaciones en cuanto a accesibilidad }\end{array}$ & 29/03/2006 \\
\hline & $\begin{array}{l}\text { Real Decreto 505/2007, de } 20 \text { de abril, por el que se aprueban las condiciones } \\
\text { básicas de accesibilidad y no discriminación de las personas con discapacidad } \\
\text { para el acceso y utilización de los espacios públicos urbanizados y } \\
\text { edificaciones }\end{array}$ & $12 / 05 / 2007$ \\
\hline & $\begin{array}{l}\text { Real Decreto } 173 / 2010 \text {, de } 19 \text { de febrero, por el que se modifica el Código } \\
\text { Técnico de la Edificación en materia de accesibilidad y no discriminación de las } \\
\text { personas con discapacidad }\end{array}$ & $12 / 03 / 2010$ \\
\hline Autonómica & $\begin{array}{l}\text { Decreto } 217 / 2001 \text {, de } 30 \text { de agosto, por el que se aprueba el Reglamento de } \\
\text { Accesibilidad y Supresión de Barreras }\end{array}$ & $05 / 12 / 2001$ \\
\hline Municipal & $\begin{array}{l}\text { Ordenanza para la supresión de barreras arquitectónicas (Pleno ayuntamiento } \\
\text { definitivo 07/02/1995) }\end{array}$ & $16 / 03 / 1995$ \\
\hline
\end{tabular}

Fuente: elaboración propia.

puede incluirse cualquier nota que resulte necesaria u oportuna para aportar más información al análisis del requisito.

3. Observaciones adicionales: En el último apartado se aclaran algunos requisitos, aportando la información necesaria para la comprensión de dicha condición.

La ficha se ha elaborado en base a documentación técnica elaborada por diferentes autores (Fundación ONCE, 20I I; Juncà, 20I I; Ministerio de Vivienda, 20II), a documentos legales y normativa existente en materia de accesibilidad, desde un rango estatal hasta municipal, detallada en la Tabla 3 .

Todos los requisitos identificados se han clasificado como críticos (obligatorios por ley y limitantes para una persona con discapacidad física) o no críticos (obligatorios según ley, pero que no limitan significativamente la autonomía de una persona con discapacidad física para utilizar el transporte público).

\subsection{Validación de cuestionario}

Finalizadas las fichas y antes de proceder con el trabajo de campo, se procedió a la verificación con el objeto de comprobar que la ficha diseñada era adecuada y contenía los parámetros necesarios.

Esta verificación se hizo en dos fases. En una primera fase se consultó a profesores y técnicos de la Universidad de Valladolid y a personal técnico y usuarios de entidades de personas con discapacidad física y orgánica agrupadas en Cocemfe Castilla y León. (www.cocemfecyl.es), organización cuya misión principal es mejorar la calidad de vida y la atención integral de las personas con discapacidad ${ }^{\mathrm{I2}}$.

En una segunda fase se revisaron varias paradas tipo de autobús comprobando in situ si los parámetros de la ficha eran adecuados

I2. En su última memoria de actividades se refleja que Cocemfe Castilla y León ha prestado atención a I 623 personas con discapacidad en sus diversos servicios, entre los que se incluyen el asesoramiento sobre sus derechos en diferentes ámbitos, entre ellos la accesibilidad universal. 
y se habían cumplido los requisitos de diseño (sencilla, comprensible, esquemática, objetiva).

Tras la validación de la ficha se elaboraron las plantillas para un adecuado tratamiento estadístico de los datos recogidos en la fase de trabajo de campo.

\subsection{Trabajo de campo}

En esta fase se han revisado los parámetros específicos de todas las paradas de autobús de la ciudad de Valladolid (58I a diciembre de 20I5).

La mayoría de las paradas se encuentran distribuidas en el núcleo urbano, si bien por la tipología de la ciudad (casco viejo, barrios periféricos, polígonos y localidades del alfoz) existen diferencias sustanciales en la concepción, localización, distribución y equipamiento de las mismas. Desde el punto de vista del análisis no se ha realizado ninguna clasificación comprobando el cumplimiento de los diferentes criterios en cada parada.

Con los datos de todas las paradas se realizó la tabulación de los cuestionarios en las plantillas diseñadas para su posterior análisis.
Para ello se ha contado con un equipo formado por cuatro personas durante un periodo de tres meses para la recogida de datos y dos meses para la tabulación y análisis.

\section{Resultados}

En la Tabla 4 se detallan los 23 requisitos analizados. Cada requisito dispone de un identificador único en función del grupo en el que se encuentra: CA (Crítico acceso a parada), CP (Crítico parada), NCA (No crítico acceso a parada), NCP (No crítico parada).

\subsection{Requisitos críticos}

El 84,5 I \% de las paradas de autobús de la ciudad de Valladolid cumplen la totalidad de los requisitos críticos en el acceso a la parada (CA).

En el Gráfico 2 se muestran los resultados de este grupo.

\section{Gráfico 2. Resultados requisitos críticos acceso a parada (CA)}

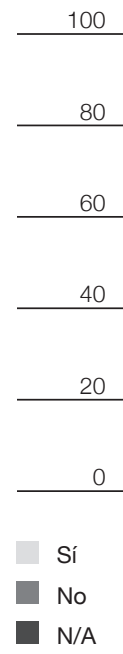

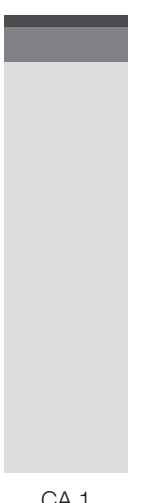

CA.1

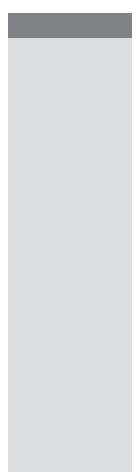

CA.2

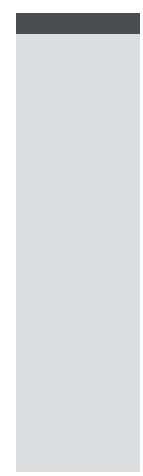

CA.3

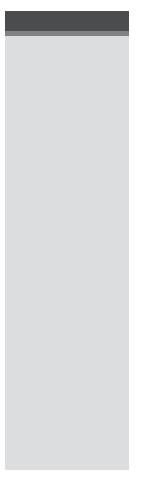

CA.4

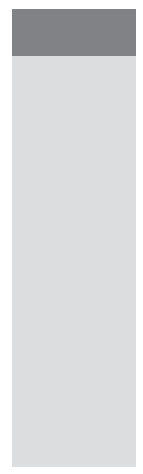

CA.5

Fuente: elaboración propia. 


\begin{tabular}{|c|c|}
\hline Identificador & Requisito \\
\hline CA.1 & Desniveles salvados por rampas (no escalones). \\
\hline CA.2 & Pavimentos sin piezas o elementos sueltos. Pavimento no deslizante, continuo y compacto. \\
\hline CA.3 & Pendiente de acera adecuada. \\
\hline CA.4 & Anchura de acera suficiente. \\
\hline CA.5 & Existencia de rebajes o vados a una distancia menor de 100 metros. \\
\hline CP.1 & $\begin{array}{l}\text { Protección inicio/fin de parada (calzada) con elementos rígidos (que eviten la invasión de } \\
\text { vehículos a la misma). }\end{array}$ \\
\hline CP.2 & Existencia de marquesina. \\
\hline CP.3 & Acceso marquesina lateral/central, paso mínimo 0,90 metros. \\
\hline CP.4 & $\begin{array}{l}\text { Anchura libre en marquesina: } 1,50 \text { metros hasta } 25 \text { centímetros de altura y } 1,35 \text { metros hasta } \\
2,10 \text { metros y altura libre } 2,10 \text { metros. }\end{array}$ \\
\hline CP.5 & $\begin{array}{l}\text { Señalización cerramiento transparente o traslúcido, } 2 \text { bandas horizontales de } 5-10 \\
\text { centímetros entre } 0,70-0,80 \text { metros y } 1,40-1,70 \text { metros color vivo. }\end{array}$ \\
\hline CP.6 & Altura libre 2,10 metros. \\
\hline CP.7 & Al menos un apoyo isquiático y un asiento. \\
\hline NCA.1 & Rejas, rejillas y tapas de registro enrasadas con pavimento. \\
\hline NCA.2 & Alcorques cubiertos o enrasados con el pavimento. \\
\hline NCP.1 & $\begin{array}{l}\text { Franja de pavimento tacto visual de } 1,20 \text { metros de ancho, perpendicular al sentido de la } \\
\text { marcha y desde bordillo hasta fachada y franja tacto visual mínimo } 40 \text { centímetros junto } \\
\text { bordillo. }\end{array}$ \\
\hline NCP.2 & Caracteres de identificación de línea altura 14 centímetros color contrastado. \\
\hline NCP.3 & Existencia de poste identificación de línea. \\
\hline NCP.4 & Información de la línea y denominación en sistema Braille en poste de identificación de línea. \\
\hline NCP.5 & $\begin{array}{l}\text { Información de identificación, denominación y esquema recorrido en sistema Braille en } \\
\text { marquesina. }\end{array}$ \\
\hline NCP.6 & Existencia de reposabrazos en asientos extremos de marquesina. \\
\hline NCP.7 & Altura de asiento 0,45 centímetros desde suelo +-2 centímetros en marquesina \\
\hline NCP.8 & Pantalla de información de situación de autobuses. \\
\hline NCP.9 & Pantalla de información de situación de autobuses con sistema sonoro. \\
\hline
\end{tabular}

Fuente: elaboración propia.

La infraestructura de la ciudad de Valladolid permite el acceso a las paradas ya que el $90,02 \%$ de las paradas tienen rampas y vados para salvar escalones. En las paradas ubicadas en las afueras de Valladolid (líneas que unen el centro de la ciudad con núcleos cercanos como Simancas o Puente Duero) no es posible aplicar este requisito, ya que no existe ninguna acera.

El pavimento de la parada $(94,84 \%)$ es adecuado, independientemente del tipo de pavimento utilizado, con diferentes tamaños, colores y distinto material. Estas poseen solados sin piezas o elementos sueltos, no deslizantes, continuos y compactos. Las paradas con el solado inadecuado coinciden con aquellas que se encuentran ubicadas en las afueras, (donde no existe acera y el pavimento es arena) o incluso en los polígonos industriales, donde la persona con discapacidad física tendría que cruzar una zona ajardinada para poder acceder al autobús. 
La pendiente de las aceras es la adecuada, únicamente se ha detectado una parada, en la cual la pendiente supera los valores definidos (menor o igual al $4 \%$ en el sentido de la marcha y al $2 \%$ en el sentido transversal. Un $4,82 \%$ no han podido evaluarse al ser paradas sin acera y en su lugar aparecen por ejemplo zonas ajardinadas y no cuidadas que impiden el acceso a personas con discapacidad física.

Las paradas con acera de anchura mínima I, 50 metros (valor considerado suficiente según la Ordenanza municipal de Valladolid), se corresponden con un 94, I $5 \%$ del total, como en los casos anteriores algunas paradas no han podido ser evaluadas al no existir acera en estas.

La distancia existente desde una parada de autobús hasta los vados o rebajes tiene una gran importancia para asegurar que una persona con discapacidad física pueda llegar a dicha parada recorriendo un trayecto de una distancia adecuada, un $88,98 \%$ de las paradas cumplen con el requisito, es decir, existen rebajes o vados que distan de las paradas menos de 100 metros, sin embargo 64 paradas no cumplen. Principalmente son las que se encuentran situadas en los polígonos industriales o en los trayectos hacia Simancas y Puente Duero.
En relación a los requisitos críticos parada (CP), únicamente el I 7,56\% de las paradas cumplen con todos los requisitos, todos los casos se encuentran en paradas que no poseen marquesina.

En el Gráfico 3 se presentan los resultados de este grupo.

En este aspecto un requisito crítico para las personas con discapacidad física es la protección de la parada con elementos rígidos que eviten invasión de vehículos a la misma, un 47,68 \% de las paradas disponen de este tipo de elementos.

Por otro lado, más de la cuarta parte de las paradas $(26,5 \mathrm{I} \%)$ no tiene ninguna protección o le falta alguna en el inicio o en el fin de la misma, con lo cual cualquier vehículo puede estacionar en ese espacio, imposibilitando el acceso del autobús y por lo tanto no permitiendo el acceso de personas con alguna discapacidad física al medio de transporte.

Las paradas en las que no se puede aplicar este requisito $(25,82 \%)$, es porque debido a su ubicación (no hay ningún espacio o aparcamiento habilitado para que pare el autobús), es imposible colocar elementos rígidos.

\section{Gráfico 3. Resultados requisitos críticos parada (CP)}

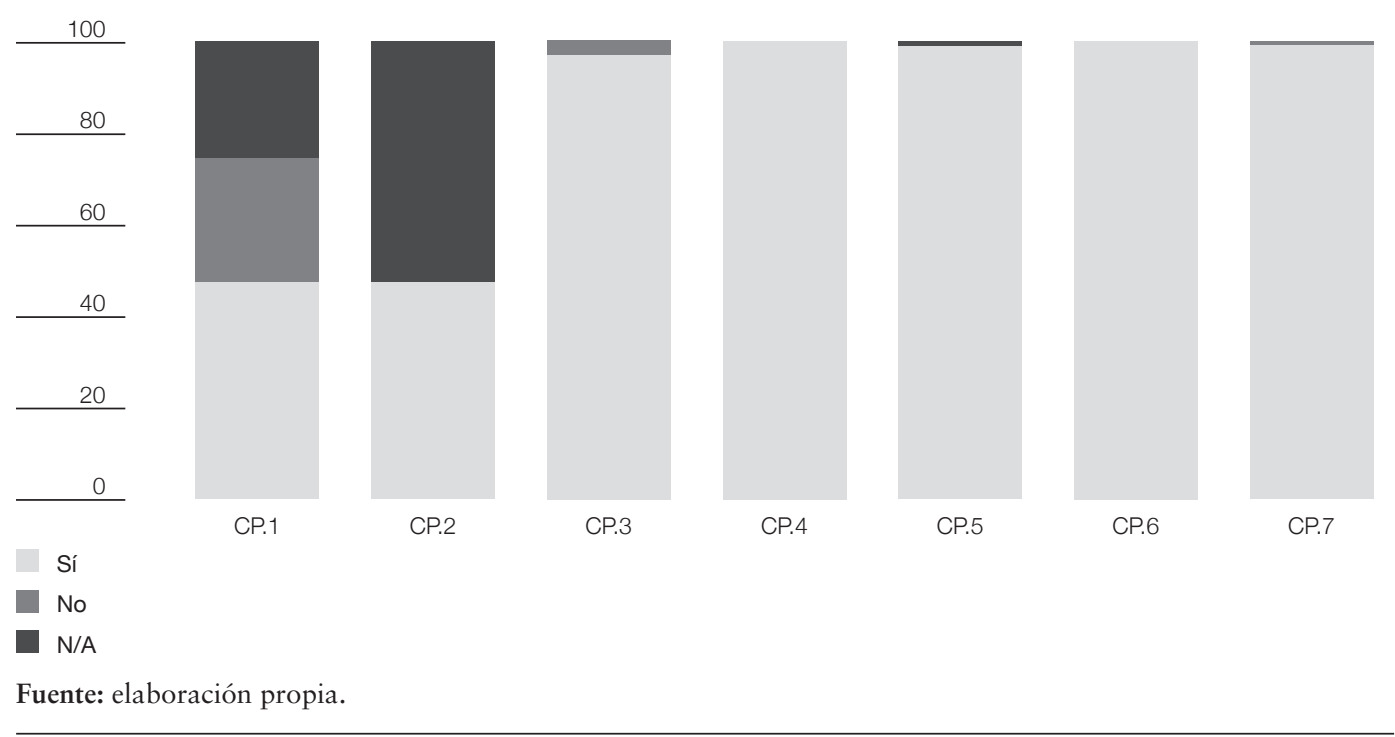


En relación a la existencia de las marquesinas en las paradas, la legislación no obliga a la existencia de una marquesina en cada parada, pero debe reflejarse que para una persona con discapacidad física la existencia de la misma hace la espera más cómoda y confortable además de proteger de las inclemencias meteorológicas. En el caso de que la parada posea marquesina esta sí debe cumplir los requisitos identificados.

Actualmente casi la mitad de las paradas de la ciudad de Valladolid poseen marquesina $(47,50 \%)$, en estas paradas el grado de cumplimiento de los requisitos relativos a la marquesina es muy alto. Accesos, dimensiones mínimas, señalización de cerramiento transparente y existencia de al menos un asiento y un apoyo isquiático, han obtenido un porcentaje muy elevado (prácticamente el ı००\%) salvo en el requisito "señalización de cerramiento transparente" que no se cumple en ninguno de los casos analizados ya que la señalización (cuando existe) es una línea pequeña de círculos amarillos contrario a lo expresado en la normativa.

\subsection{Requisitos no críticos}

Los requisitos no críticos han sido calificados como tales por los expertos consultados en función del criterio de no limitar significativamente la autonomía de una persona con discapacidad física para la utilización del transporte público, lo que no impide que sea obligatorio su cumplimiento por la normativa vigente.

El 67,8I \% de las paradas de autobús de la ciudad de Valladolid cumplen la totalidad de los requisitos no críticos en el acceso a la parada (NCA).

En el Gráfico 4 se muestran los resultados de este grupo.

Un 92,08\% de las paradas tienen las tapas de registros, rejillas y rejas enrasadas con el pavimento, mientras que en el porcentaje restante no ha sido posible el estudio al no

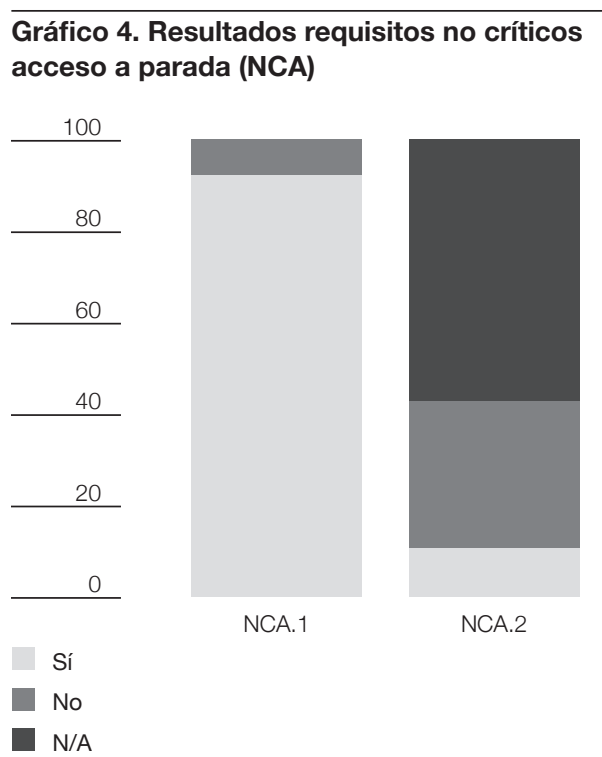

Fuente: elaboración propia.

existir ninguna, por lo tanto puede afirmarse que este requisito se cumple en todas las paradas que posean estos elementos.

Sin embargo el porcentaje de los alcorques cubiertos o enrasados con el pavimento es mucho más bajo (ıо,33\%), además más de la mitad de las paradas no disponen de estos elementos $(57,49 \%)$, por lo que el requisito se cumple en un $24,29 \%$ del total que tienen alcorques.

En relación a los requisitos no críticos en la parada (NCP), ninguna de las 58 I de la ciudad de Valladolid cumple todos los requisitos.

En el Gráfico 5 se presentan los resultados de este grupo.

Nueve de cada diez paradas no disponen de una franja de pavimento tacto visual de anchura I,20 metros en sentido perpendicular al sentido de la marcha señalizando la parada del autobús como indica la normativa vigente (aspecto crítico para personas con discapacidad visual). La mayoría de las paradas que cumplen este requisito han sido reformadas últimamente. 


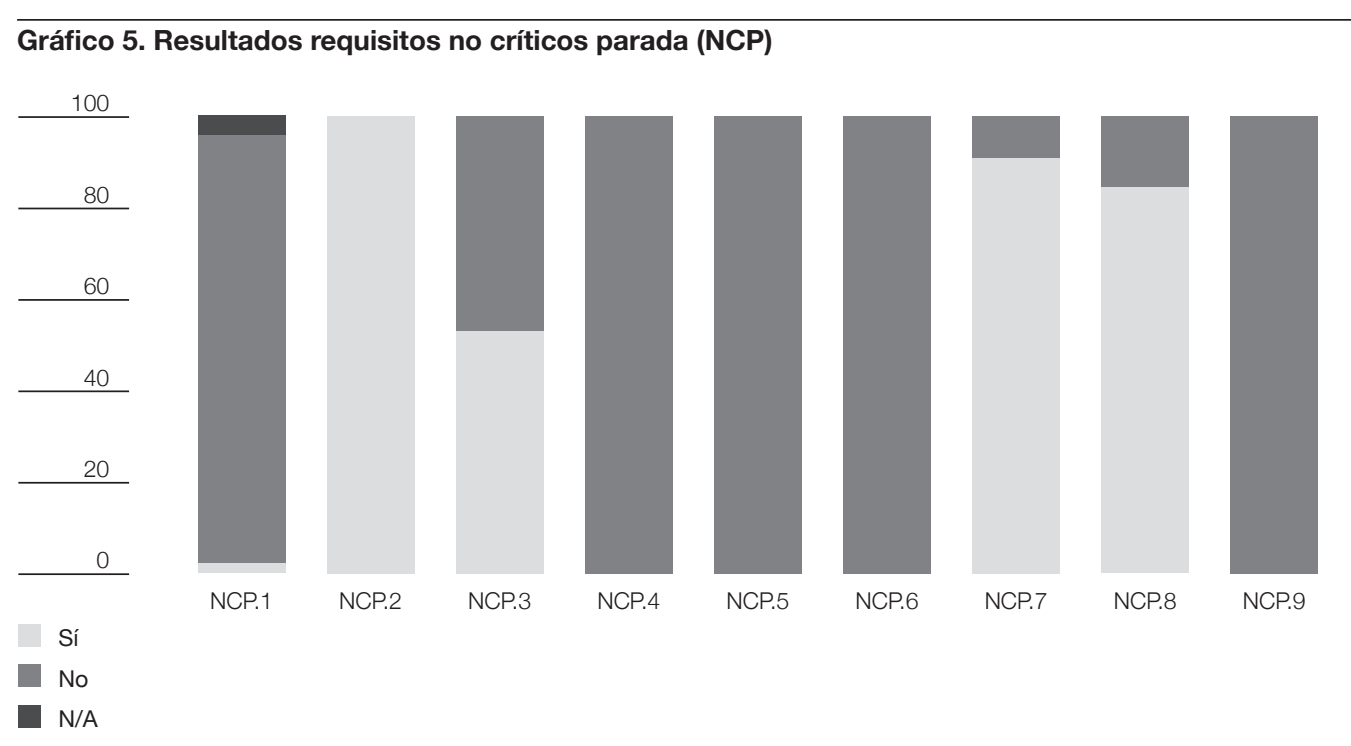

Fuente: elaboración propia.

Los últimos requisitos analizados hacen referencia a la existencia de poste de identificación de línea, existencia de marquesina y pantalla de información de autobuses. En el caso que existan tienen que cumplir además otros requisitos como la información en sistema Braille en el poste o el dispositivo sonoro para la pantalla de información de autobuses.

En prácticamente todas las paradas de autobús aparece la identificación de la línea con letras y números de una altura mayor a I4 centímetros y color contrastado, únicamente en tres paradas no aparece esa información y una en la que no se puede aplicar, que se corresponde con la parada del estadio de fútbol José Zorrilla que no posee ni poste ni marquesina.

En la Tabla 5 se indican las paradas que tienen poste y/o marquesina del total de las 58 I de la ciudad de Valladolid.

Ninguna de las marquesinas tiene la información, denominación y esquema del recorrido en sistema Braille, tampoco poseen reposabrazos en ningún asiento (ni central ni extremo), el requisito de la altura del asiento sí se cumple en la mayoría de casos (90,22\%).

\begin{tabular}{|c|c|c|c|}
\hline \multicolumn{4}{|c|}{ Paradas (Total 581) } \\
\hline $\begin{array}{l}\text { Con } \\
\text { poste }\end{array}$ & $\begin{array}{c}\text { Con } \\
\text { marquesina }\end{array}$ & $\begin{array}{l}\text { Con } \\
\text { ambos }\end{array}$ & Sin nada \\
\hline 309 & 276 & 5 & 1 \\
\hline
\end{tabular}

Fuente: elaboración propia.

El $16,35 \%$ de las paradas disponen de pantalla de información de la situación de autobuses, pero ninguna de ellas posee un dispositivo sonoro para la información de personas con discapacidad auditiva.

\section{Conclusiones}

Se ha definido un método para evaluar la accesibilidad a los medios de transporte público para las personas con discapacidad física, pero considerando la utilización del transporte público de manera autónoma, no el cumplimiento de la normativa en su totalidad. 
En este estudio se ha analizado la cadena de accesibilidad (itinerario, instalación, medio) para el transporte público de autobús, si bien puede ser ampliado a otros medios de transporte como el ferrocarril y el taxi.

La ciudad de Valladolid ha realizado avances importantes en la adaptación de su transporte público, aunque no se cumplan aún todos los requisitos de accesibilidad establecidos en la normativa vigente.

La totalidad de los requisitos definidos como críticos (tanto en acceso como en la parada) se cumplen en un bajo porcentaje $(\mathrm{I} 4,46 \%)$. Debe señalarse que los requisitos críticos de acceso a la parada se cumplen en un porcentaje muy alto $(92 \%)$ lo que refleja que las aceras, los pavimentos, vados y rampas de la ciudad de Valladolid están preparados para que una persona con discapacidad física acceda de manera autónoma a la parada. Sin embargo los requisitos críticos en la parada son los que penalizan el grado de cumplimiento total. Estos resultados pueden mejorar notablemente incidiendo solamente en dos aspectos críticos:

I. Actuando sobre las protecciones necesarias en el inicio y final de las paradas, con el objeto de evitar que otros vehículos invadan el espacio destinado utilizado por el autobús para parar. Se ha detectado que los autobuses no pueden estacionar en las propias paradas correctamente porque están invadidas por otros vehículos, esto supone que el autobús tenga que parar en otro lugar no habilitado para ello y, por lo tanto, que las personas con algún tipo de discapacidad física no puedan acceder al mismo. La existencia de protecciones en las paradas de autobús para que no ocurra este problema no llega al $50 \%$.

2. En el caso de las marquesinas, señalizar adecuadamente sus cerramientos transparentes de vidrio en base a la normativa vigente (no como se realiza en la actualidad), con dos bandas horizontales de cinco a diez centímetros situadas a una altura de entre 0,70 y $\circ, 8 \circ$ metros y entre
I,40 y I,70 metros en color vivo. Esta señalización evita que la persona pueda chocar con el cerramiento al distinguirla frente al cristal de la marquesina. El resto de requisitos aplicables a la marquesina alcanzan valores de cumplimiento altos (entre un $96,74 \%$ y un Io० \%).

Los requisitos no críticos no se cumplen en su totalidad en ninguna parada. Como se ha detallado en el estudio estos requisitos no son esenciales para que una persona con discapacidad física pueda acceder a la parada de autobús o permanecer en ella, presentando un mayor inconveniente para personas con otro tipo de discapacidad, como por ejemplo discapacidad visual, sin obviar en todo caso que su cumplimiento está reflejado en la normativa vigente.

Únicamente se cumplen dos requisitos en más de un $90 \%$, altura de los caracteres de identificación de línea mayor de I 4 centímetros y color contrastado y la altura de los asientos en las paradas que disponen de ellos.

El incumplimiento de los requisitos no críticos, se debe principalmente a los que están incluidos en la parada ya que:

I. No existe la franja de pavimento tacto visual ni en sentido perpendicular a la parada ni junto al bordillo en más de un $92 \%$ de paradas.

2. En el Ioo\% de las paradas no existe información en sistema Braille ni en postes ni en marquesinas, no existe ninguna parada con marquesina en la que existan reposabrazos en asientos, ni tampoco existe dispositivo sonoro alguno en las que tienen una pantalla de información de la situación de autobuses.

Sin embargo, en los requisitos no críticos de acceso a la parada, se obtienen unos resultados muy distintos entre sí. El resultado es más favorable en las paradas cuyas aceras poseen rejas, rejillas y tapas de arqueta, ya que están enrasadas con el pavimento, sin embargo 
en las paradas cuyas aceras están provistas de alcorques, únicamente un 24,29\% están cubiertos o enrasados con el pavimento.

Para finalizar, y como trabajo futuro, nos planteamos la extensión de este estudio utilizando la metodología aquí definida a otros medios de transporte público como el ferrocarril y el taxi, y al estudio de otras localidades de la comunidad autónoma de Castilla y León. 


\section{Referencias bibliográficas}

Aarhaug, J. y Elvebakk, B. (201 5): "The impact of Universally accessible public transport-a before and after study". Transport Policy, 44: I 43 -I 5 O.

Ararteko (20I I): Diagnóstico de accesibilidad del sistema de transporte público en la CAPV (en línea). <http://www.ararteko.net/RecursosWeb/ DOCUMENTOS/I/I_2264_3.pdf>, acceso 22 de octubre de 2015 .

Ayuntamiento de Valladolid (20I I): I Plan Municipal de Accesibilidad (en línea). $<$ http://www.valladolid.es/es/gente/personasdiscapacidad/servicios/i-plan-municipalaccesibilidad.ficheros/270 I I-MAQUETA \% 20 DEL \% 20PLAN\% 2०EN\% 2०PDF.pdf $>$, acceso I9 de junio de 20 I 6 .

Barnes, C. y Mercer, G. (2005): “Disability, work, and welfare challenging the social exclusion of disabled people". Work, Employment \& Society, I9 (3): 527-545.

Casas, I. (2007): "Social exclusion and the disabled: An accessibility approach". The Professional Geographer, 59 (4): 463-477.

Castilla y León. Decreto 2 I7/200I, de 30 de agosto, por el que se aprueba el Reglamento de accesibilidad y supresión de barreras, Boletín Oficial de Castilla y León, 4 de septiembre de 200I, núm. I72, pp. I2984-I30I4.

Castilla y León. Ley 3/1998, de 24 de junio de accesibilidad y supresión de barreras de Castilla y León, Boletín Oficial de Castilla y León, I de julio de 2008, núm. I23, pp. 60I2-603I.

Corbacho, A. et al. (2003): "Sociología de la discapacidad: exclusión e inclusión social de los discapacitados". Revista del Ministerio de Trabajo e Inmigración, 45: 77-106.

Díaz Velázquez, E. (2010): “Ciudadanía, identidad $y$ exclusión social de las personas con discapacidad". Politica y sociedad, 47 (I): II 5-I 35
España. Constitución Española, Boletín Oficial del Estado, núm. 3 I I, de 29 de diciembre de 1978 , pp. 293I3-29424.

España. Instrumento de Ratificación de la Convención sobre los derechos de las personas con discapacidad, hecho en Nueva York el I3 de diciembre de 2006, Boletín Oficial del Estado, 2I de abril de 2008, núm. 96, pp. 20648-20659.

España. Ley I3/I982, de 7 de abril, de integración social de los minusválidos, Boletín Oficial del Estado, 30 de abril de 1982, núm. Io3, pp.IIIO6-IIII2.

España. Ley 26/20I I, de I de agosto, de adaptación normativa a la Convención Internacional sobre los Derechos de las Personas con Discapacidad, Boletín Oficial del Estado, 2 de agosto de 20I I, núm. I 84, pp. $87478-87494$.

España. Ley 39/2006, de I4 de diciembre, de Promoción de la Autonomía Personal y Atención a las personas en situación de dependencia, Boletín Oficial del Estado, I 5 de diciembre de 2006, núm. 299, pp. 44I4244I 56 .

España. Ley 5 I $/ 2003$, de 2 de diciembre, de igualdad de oportunidades, no discriminación y accesibilidad universal de las personas con discapacidad, Boletín Oficial del Estado, 3 de diciembre de 2003, núm. 289, pp.43I8743195.

España. Real Decreto Legislativo I/20I3, de 29 de noviembre, por el que se aprueba el Texto Refundido de la Ley General de derechos de las personas con discapacidad y de su inclusión social, Boletín Oficial del Estado, 3 de diciembre de 2013, núm. 289, pp. $95635^{-}$ 95673 .

España. Real Decreto I73/20Io, de I9 de febrero, por el que se modifica el Código Técnico de la Edificación, aprobado por el Real Decreto 3 I4/2006, de I7 de marzo, en materia de 
accesibilidad y no discriminación de las personas con discapacidad, Boletín Oficial del Estado, i de marzo de 20Io, núm. 6I, pp. 245 I0-24562.

España. Real Decreto I 276/20I I, de I6 de septiembre, de adaptación normativa a la Convención Internacional sobre los derechos de las personas con discapacidad, Boletín Oficial del Estado, I 7 de septiembre de 20I I, núm. 224, pp.98872-98879.

España. Real Decreto I 544/2007, de 23 de noviembre, por el que se regulan las condiciones básicas de accesibilidad y no discriminación para el acceso y utilización de los modos de transporte para personas con discapacidad, Boletín Oficial del Estado, 4 de diciembre de 2007, núm. 290, pp. 49948- 49975.

España. Real Decreto 505/2007, de 20 de abril, por el que se aprueban las condiciones básicas de accesibilidad y no discriminación de las personas con discapacidad para el acceso y utilización de los espacios públicos urbanizados y edificaciones, Boletín Oficial del Estado, I I de mayo de 2007, núm. II3, pp. 20384-20390.

España. Real Decreto 3 I4/2006, de I7 de marzo, por el que se aprueba el Código Técnico de la Edificación, Boletín Oficial del Estado, 28 de marzo de 2006, núm. 74, pp. I I8I6-I I83 I.

Ferreira, M.A. (2008): “La construcción social de la discapacidad: habitus, estereotipos y exclusión social”. Nómadas. Revista Crítica de Ciencias Sociales y Jurídicas, I7 (I): 22 I-232.

Field, M.J. y Jette, A. (eds.) (2007): The future of disability in America. Washington, DC: National Academies Press.

Fundación ONCE (2OII): Manual de accesibilidad para técnicos municipales (en línea). <http:// www.fundaciononce.es/sites/default/files/docs/ ManualAccesibilidad_2.pdf $>$, acceso I3 de mayo de 2016.

Huete, A. (2013): "La exclusión de la población con discapacidad en España. Estudio específico a partir de la Encuesta Social Europea”. Revista Española de Discapacidad, I (2): 7-24.
Huete, A. (2OI2): "La discapacidad como factor de exclusión social. Evidencias empíricas desde una perspectiva de derechos" (en línea). <http:// gredos.usal.es/jspui/bitstream/I0366/I 2 I I 74/3/ INICO_HueteGarciaAgustin_Tesis.pdf>, acceso 20 de enero de 2016.

Imserso (2013): Base estatal de datos de personas con valoración del grado de discapacidad (en línea). <http://imserso.es/InterPresentr/groups/ imserso/documents/binario/bdepcd_20I3.pdf>, acceso I 6 de diciembre de 20 I 5 .

Imserso (2004): Atención a las personas en situación de dependencia en España. Libro blanco (en línea). <http://www.dependencia. imserso.es/InterPresent I/groups/imserso/ documents/binario/libroblanco.pdf $>$, acceso I9 de junio de 2016 .

INE (2015): Padrón. Población por municipios (en línea). <http://www.ine. es/jaxi/menu.do?type=pcaxis\&path=/t2o/ e245/\&file=inebase>, acceso I 6 de enero de 2016.

INE (2008): Encuesta sobre discapacidades, autonomía personal y situaciones de dependencia. EDAD 2008 (en línea). <http:// www.ine.es/dyngs/INEbase/es/operacion.ht $\mathrm{m}$ ? $\mathrm{c}=$ Estadistica_C\&cid $=\mathrm{I} 254736176782 \&$ menu=resultados \&secc $=$ I 254736 I 947 I $6 \&$ i $\mathrm{dp}=\mathrm{I} 254735573 \mathrm{I} 75>$, acceso I9 de junio de 2016.

Juncà, J.A. (20I I): "Diseño de transporte accesible", en Hernandez, J.: Accesibilidad Universal y Diseño para Todos. Arquitectura y Urbanismo. Madrid: Fundación ONCEFundación Arquitectura COAM.

Juncà, J.A. (2006): Diseño Universal: Factores Clave para la Accesibilidad Integral. $2^{a}$ ed., Madrid: Cocemfe.

Lucas, K. (2OI2): “Transport and social exclusion: Where are we now?”. Transport policy, 20: IO5-II3.

Ministerio de Vivienda (20I I): Accesibilidad en los espacios públicos urbanizados (en línea). <http://www.fomento.gob. es/NR/rdonlyres/EC23 $3 \mathrm{~F}_{7}$ I-B5EB4482-8E 3 D-IoB40D25 I397/I I6390/ 
ACCESEspaPublicUrba.pdf $>$, acceso i I de marzo de 2016.

ONU (2006): Convención sobre los Derechos de las Personas con Discapacidad. Nueva York: Organización de las Naciones Unidas.

Preston, J. (2009): “Epilogue: Transport policy and social exclusion-Some reflections". Transport policy, I6 (3): I40-I42.

Preston, J. y Rajé, F. (2007): “Accessibility, mobility and transport-related social exclusion”. Journal of Transport Geography, I 5 (3): I 5 I-I 60.
Priya, T. y Uteng, A. (2009): "Dynamics of transport and social exclusion: Effects of expensive driver's license". Transport policy, I6 (3): I30-I39.

Stanley, J. et al. (2010): Social Exclusion and the Value of Mobility (en línea). <http://sydney.edu. au/business/_data/assets/pdf_file/oo04/729I3/ itls-wp-Io-I 4.pdf>, acceso 20 de enero de 2016.

Valladolid. Plan Integral de Movilidad Urbana Ciudad de Valladolid (PIMUVA), Boletín Oficial de la Provincia de Valladolid, I de marzo de 2005, núm. 49, pp. 9-60. 